\title{
methyl-ATAC-seq measures DNA methylation at accessible chromatin
}

\author{
Roman Spektor, ${ }^{1}$ Nathaniel D. Tippens, ${ }^{2}$ Claudia A. Mimoso, ${ }^{3}$ and Paul D. Soloway ${ }^{4,5}$ \\ ${ }^{1}$ Department of Molecular Biology and Genetics, Field of Genetics, Genomics, and Development, Cornell University, Ithaca, New York \\ 14853, USA; ${ }^{2}$ Tri-Institutional Training Program in Computational Biology and Medicine, Cornell University, Ithaca, New York \\ 14853, USA; ${ }^{3}$ College of Agricultural and Life Sciences, Cornell University, Ithaca, New York 14853, USA; ${ }^{4}$ College of Veterinary \\ Medicine, Department of Biomedical Sciences, Cornell University, Ithaca, New York 14853, USA; ${ }^{5}$ College of Agriculture and Life \\ Sciences, Division of Nutritional Sciences, Cornell University, Ithaca, New York 14853, USA
}

\begin{abstract}
Chromatin features are characterized by genome-wide assays for nucleosome location, protein binding sites, three-dimensional interactions, and modifications to histones and DNA. For example, assay for transposase accessible chromatin sequencing (ATAC-seq) identifies nucleosome-depleted (open) chromatin, which harbors potentially active gene regulatory sequences; and bisulfite sequencing (BS-seq) quantifies DNA methylation. When two distinct chromatin features like these are assayed separately in populations of cells, it is impossible to determine, with certainty, where the features are coincident in the genome by simply overlaying data sets. Here, we describe methyl-ATAC-seq (mATAC-seq), which implements modifications to ATAC-seq, including subjecting the output to BS-seq. Merging these assays into a single protocol identifies the locations of open chromatin and reveals, unambiguously, the DNA methylation state of the underlying DNA. Such combinatorial methods eliminate the need to perform assays independently and infer where features are coincident.
\end{abstract}

[Supplemental material is available for this article.]

Active promoters, enhancers, and other gene regulatory sequences are typically bound by sequence-specific transcription factors (TFs), free of nucleosomes, and these facilitate transcription. Such regulatory sequences can be identified by methods that detect nucleosome-depleted regions (NDRs), including DNaseseq, which identifies NDRs by their hypersensitivity to DNase I (Thurman et al. 2012); FAIRE-seq, which identifies NDRs according to their reduced protein content (Gaulton et al. 2010); and ATAC-seq, which identifies NDRs based on their increased accessibility to Tn5 transposase integration, and accordingly are called Transposase hypersensitive sites (THS) (Buenrostro et al. 2013). There is considerable agreement among the regions identified by each assay. ATAC-seq has received further use recently owing to its simplified workflow, reduced material requirements, and lower background signals. Additional advancements such as OmniATAC (Corces et al. 2017) and Fast-ATAC (Corces et al. 2016) have further improved the utility of ATAC-seq.

DNA within NDRs may have different modification states, including methylation at the fifth carbon of Cytosine $(5 \mathrm{mC})$, and oxidized derivatives. In the mammalian genome, most $5 \mathrm{mC}$ is found at CpG dinucleotides, and is generally associated with transcriptionally inactive regions. Bisulfite sequencing (BS-seq) uses selective chemical deamination of unmodified cytosines to uracil, leaving $5 \mathrm{mC}$ unchanged. The extent of methylation at a given $\mathrm{CpG}$ in a sample is detected after amplification, sequencing, aligning reads to the genome, and then assessing the proportion of aligned reads that retained a $\mathrm{C}$ at a $\mathrm{CpG}$, diagnostic of methylation, versus a $\mathrm{T}$, which reports an unmethylated residue.

Two features of BS-seq significantly increase costs compared to routine sequencing assays. First, bisulfite treatment reduces

Corresponding author: soloway@cornell.edu

Article published online before print. Article, supplemental material, and publication date are at http://www.genome.org/cgi/doi/10.1101/gr.245399.118. the yield and complexity of DNA libraries, resulting in fewer reads uniquely aligning to the genome. Second, to reliably quantify the extent of methylation of a given $\mathrm{CpG}$ requires high read coverage. For these reasons, reduced representation bisulfite sequencing (RRBS) (Meissner et al. 2005) and derivatives (Boyle et al. 2012; Chatterjee et al. 2012; Garrett-Bakelman et al. 2015) have been used to focus analysis on CpG dense regions. However, not all gene regulatory sequences are detected by RRBS, and many regions that are detected are not regulatory.

Integrating results from assays for distinct chromatin features have defined novel categories of regulatory elements. These include bivalent promoters (Bernstein et al. 2006), enhancers (Heintzman et al. 2009), and widely observed chromatin states likely to harbor shared regulatory functions (Roadmap Epigenomics Consortium et al. 2015). In most of these studies, results from assays for single features are superimposed, and when a given locus has signals for multiple features, the features are inferred to be coincident on the same molecule. Although many inferences might be accurate, there is uncertainty inherent in such approaches, owing to the fact that samples commonly contain multiple subpopulations of cells, each with a characteristic chromatin state. Accordingly, the population-averaged results might report chromatin states found in no individual subpopulation of cells. Methods that combine assays for multiple chromatin features in a single protocol can eliminate this ambiguity for the features assayed. Here, we describe methyl-ATAC-seq (mATAC-seq), a modification of ATAC-seq that combines ATAC-seq with BS-seq, identifying the locations of open chromatin, and the methylation

(C) 2019 Spektor et al. This article is distributed exclusively by Cold Spring Harbor Laboratory Press for the first six months after the full-issue publication date (see http://genome.cshlp.org/site/misc/terms.xhtml). After six months, it is available under a Creative Commons License (Attribution-NonCommercial 4.0 International), as described at http://creativecommons.org/licenses/ by-nc/4.0/. 
state of the underlying DNA. In addition to providing more reliable assignments of chromatin states, mATAC-seq can focus DNA methylation analyses to accessible regulatory regions of the genome.

\section{Results}

Figure 1 shows the workflow and sample results for mATAC-seq. It includes two primary modifications during the transposition step of the Omni-ATAC-seq protocol: (1) Methylated oligonucleotides are loaded onto Tn5 to generate the transposome (Fig. 1A), which is then used to perform ATAC-seq (Fig. 1B); and (2) 5-methyldeoxycytosine triphosphate (5-mdCTP) is substituted for dCTP during the subsequent end repair step (Fig. 1C). These modifications protect the Nextera adapter sequences during the final step of mATAC-seq library preparation, bisulfite treatment of the tagmented DNA (Fig. 1D). Use of methylated oligonucleotides and 5-mdCTP during end repair protects cytosines in the

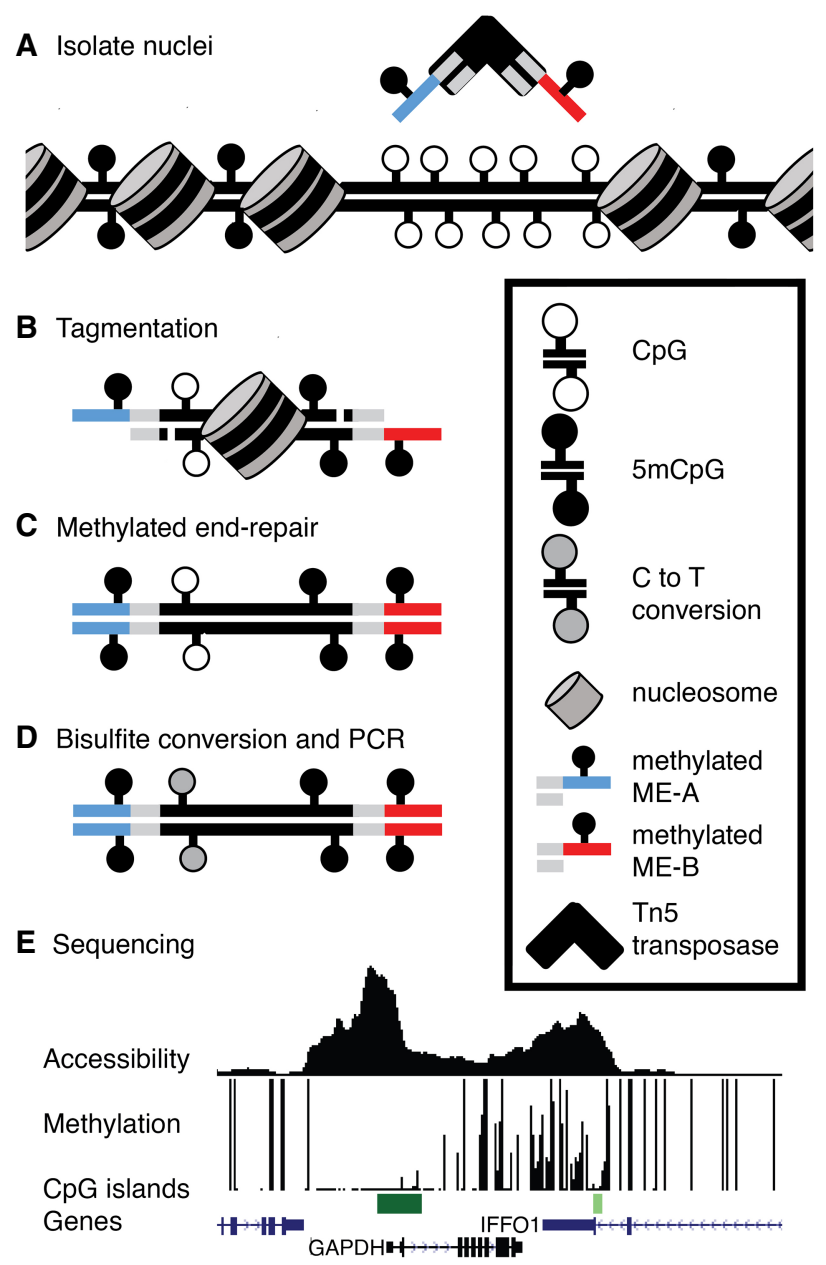

Figure 1. Overview of mATAC-seq. ( $A$ ) Tn5 carrying methylated oligonucleotides (red and blue segments) is used to $(B)$ perform tagmentation on nuclei at THS sites. (C) Tagmented DNA is end-repaired using $5 \mathrm{mdCTP}+\mathrm{dDTPs}$, purified, $(D)$ bisulfite-converted, amplified, and $(E)$ sequenced to measure DNA methylation and accessibility simultaneously. Sample data are shown for one region in HCT116 cells. Peak height in accessibility track is proportional to read abundance; bar height in methylation track is proportional to extent of methylation at CpGs. adapters from deamination caused by bisulfite treatment, which is necessary for successful PCR amplification and sequencing of the resulting libraries. Sequenced libraries provide information on both DNA methylation and Transposase hypersensitivity (Fig. 1E).

We applied mATAC-seq to nuclei prepared from HCT116 colorectal carcinoma cells. mATAC-seq reads in peaks were highly reproducible in biological replicates $\left(r^{2}=0.90\right)$ (Supplemental Fig. $\mathrm{S} 1 \mathrm{~A})$. To validate that mATAC-seq captured open chromatin domains as well as conventional methods, we compared transposase hypersensitive (THS) sites found by mATAC-seq with those we identified using the standard Omni-ATAC-seq protocol (Fig. 2AD; Corces et al. 2017). Approximately $92 \%$ of called peaks found by Omni-ATAC-seq were found by mATAC-seq (Fig. 2A). There was also strong concordance between mATAC-seq and OmniATAC-seq with respect to gene features detected by both assays, with promoter regions being the most commonly identified features (Fig. 2B). In addition, reads in peaks identified by OmniATAC-seq and mATAC-seq were well correlated (Supplemental Fig. S1A,B). Regions of greatest divergence include difficult to map regions such as repetitive elements, low complexity sequences, and simple repeat annotations (Supplemental Fig. S1C). These analyses demonstrate that mATAC-seq detects open chromatin comparably to traditional Omni-ATAC-seq, and that protocol modifications that enable subsequent bisulfite sequencing do not compromise detection of open chromatin.

To validate that mATAC-seq identified DNA methylation patterns as reliably as conventional methods, we next compared the mATAC-seq methylation data with whole-genome bisulfite sequencing (WGBS) data reported for HCT116 cells at THS sites and CpG islands (Blattler et al. 2014). DNA methylation detected by mATAC-seq replicates was highly reproducible at peaks $\left(r^{2}=0.83\right)$ and CpG islands $\left(r^{2}=0.95\right)$ (Supplemental Fig. S2A,B); methylation levels reported by mATAC-seq correlated well with levels reported by WGBS at peaks $\left(r^{2}=0.68\right)$ and CpG islands $\left(r^{2}=0.85\right)$ (Supplemental Fig. S2A,B). THS peaks identified by mATAC-seq in HCT116 were predominantly unmethylated, and this agrees with existing WGBS data (Supplemental Fig. S2C,D). Figure 2, E and F, report DNA methylation patterns assayed respectively by mATAC-seq and WGBS across gene bodies spanning from $2 \mathrm{~kb}$ $5^{\prime}$ of transcriptional start sites (TSS) to $2 \mathrm{~kb} 3^{\prime}$ of transcriptional end sites (TES). These patterns are consistent with the high correlations described above. We find these high correlations despite the fact that the assays were performed by different laboratories; also, WGBS and mATAC-seq assays are different in that mATAC-seq queries DNA methylation at open chromatin, whereas WGBS assays the entire genome, regardless of chromatin state. Our mATAC-seq data showed a reciprocal relationship between accessibility and $5 \mathrm{mC}$ density. These are in agreement with previous results from NOMe-seq (Kelly et al. 2012), which can also report sites of accessible chromatin and DNA methylation states but requires much greater sequencing depth. Both assays reveal that highly accessible chromatin is depleted of $5 \mathrm{mC}$, and that there is an abundance of methylation in less accessible chromatin over gene bodies (Fig. 2E,F). Having shown that sites of open chromatin and DNA methylation states reported by mATAC-seq, Omni-ATAC-seq, and WGBS are in agreement, we concluded that mATAC-seq can be used to simultaneously identify the locations of the genome with accessible chromatin and the methylation state of the underlying DNA. Because mATAC-seq measures accessibility and methylation in a single assay, it eliminates the inherent uncertainty about coincidence of chromatin features that can arise when ATAC and

\section{Genome Research}

www.genome.org 
mATAC-seq: DNA methylation at accessible chromatin
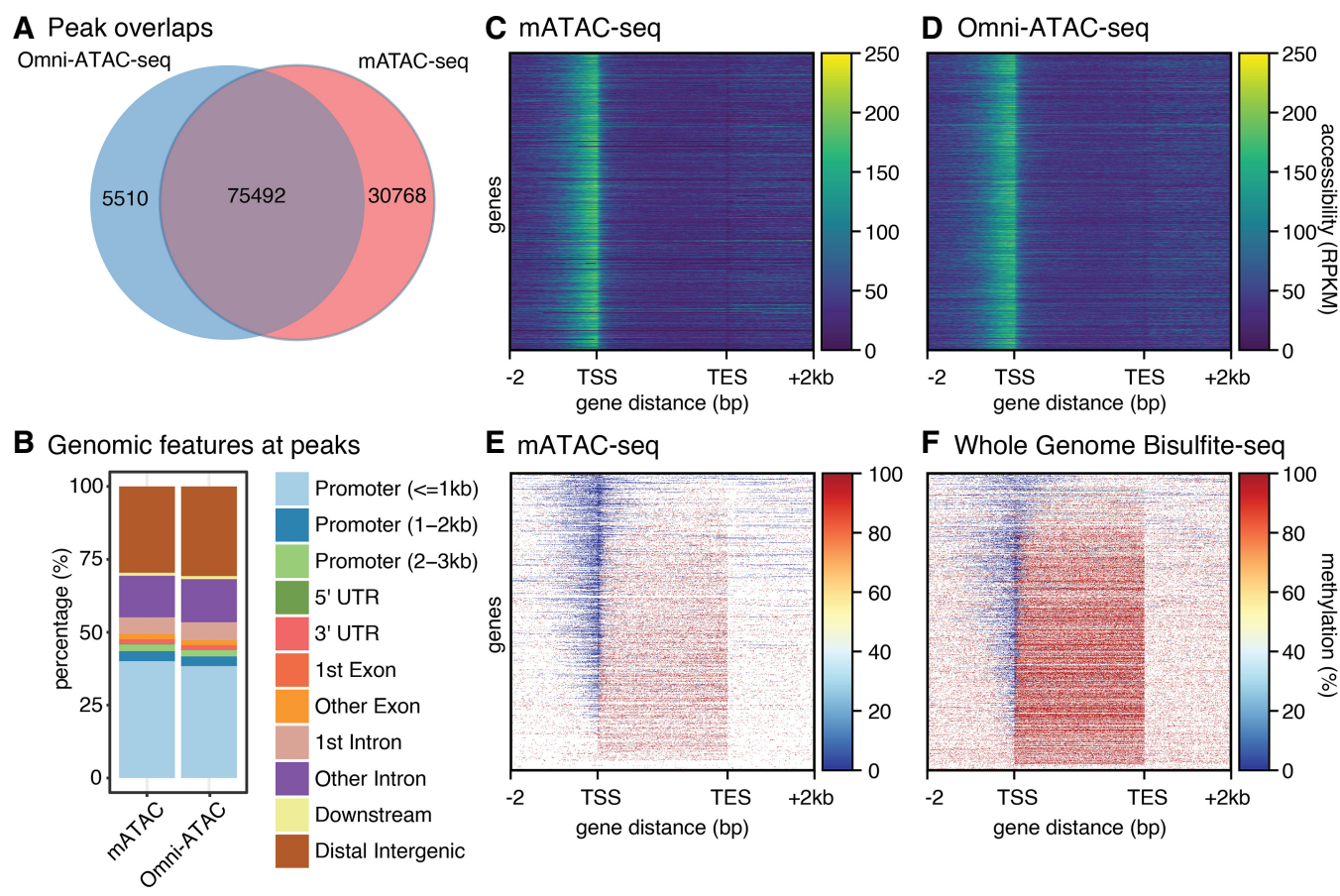

\section{E mATAC-seq}
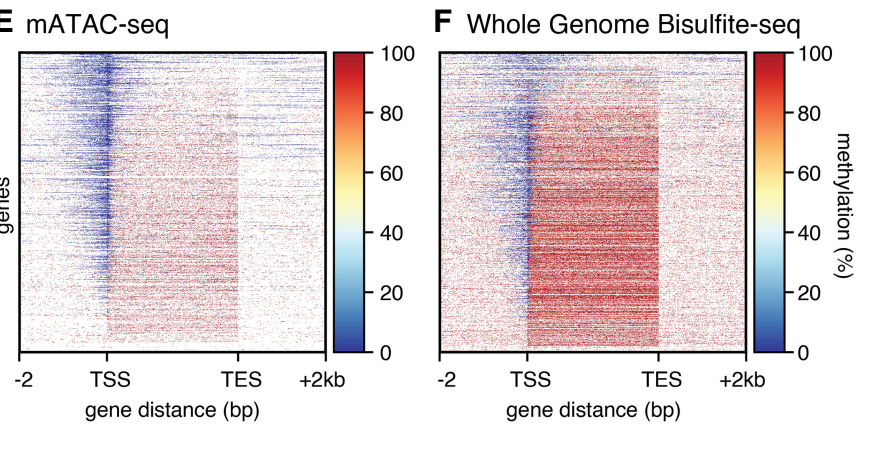

Figure 2. Comparison of methods. ( $A$ ) Omni-ATAC-seq and mATAC-seq share a majority of peaks. (B) Features at peaks are similar for mATAC-seq and Omni-ATAC-seq. $(C, D)$ Accessibility in mATAC-seq is comparable to Omni-ATAC-seq at gene bodies $\pm 2 \mathrm{~kb}, n=21,305$. $(E, F)$ Methylation reported by mATAC is comparable to WGBS at gene bodies $\pm 2 \mathrm{~kb}, n=21,305$, although WGBS includes data absent from mATAC.

bisulfite assays are performed independently and inferences are made after overlaying the two data sets, and at lower costs.

We extended our analyses of HCT116 cells, performing mATAC-seq on HCT116-derived DNMT1 and DNMT3B double knockout cells (DKO) (Rhee et al. 2002) to assess the functional significance of these methyltransferases on chromatin accessibility and methylation states in parental HCT116 cells. In DKO cells, there were 23,301 hyperaccessible sites, and 3166 hypoaccessible sites, compared to parental HCT116 cells (| $\log _{2}$ fold change $\mid>1$, $q<0.01$ ) (Fig. 3A); 16,170 THS sites observed in HCT116 cells were unchanged in DKO cells $\left(\mid \log _{2}\right.$ fold change $\left.\mid<1, q>0.8\right)$. Compared to unchanged sites, hyperaccessible sites in DKO cells were depleted of DNA methylation (Fig. 3B); these sites were enriched for ATF3, FOSL1, FOSL2, BATF, AP1, and JUNB binding motifs (Fig. 3C). These TFs were previously shown to interact more strongly to their binding motifs when unmethylated (methylminus TFs) (Yin et al. 2017). We infer that chromatin hyperaccessibility at these sites in DKO cells was attributable to enhanced binding of the methyl-minus TFs when methylation was diminished; this had the effect of limiting nucleosome deposition, thus enabling increased chromatin accessibility. Conversely, hypoaccessible sites in DKO cells were modestly depleted of DNA methylation (Fig. 3B), and enriched for SP1, NFYA, SP5, KLF9, KLF14, and KLF3 binding motifs (Fig. 3D). These TFs were previously shown to exhibit less binding when their sites were unmethylated (methylplus TFs) (Yin et al. 2017). We infer that chromatin hypoaccessibility at these sites in DKO cells was a result of reduced binding of the methyl-plus TFs when methylation was diminished, and that this led to increased nucleosome deposition and reduced chromatin accessibility. In support of this is the observation that promoters showing the greatest increases in chromatin accessibility in DKO cells were also the promoters that were most extensively hypo- methylated (Fig. 3E). These findings and conclusions are consistent with previously described mechanisms whereby TF binding can regulate nucleosome density (Zaret and Carroll 2011). These conclusions may be tempered by the fact that we are assaying methylation at accessible sequences, the same loci, when in an inaccessible state, are underrepresented in our methylation analyses.

To assess how promoter accessibility states detected by mATAC-seq relate to gene expression, we queried existing RNAseq data from HCT116 and DKO cells (Blattler et al. 2014). Promoters that were hypoaccessible in DKO cells exhibited no significant gene expression changes relative to the corresponding promoters in parental HCT116 cells. At promoters that exhibited no differences in accessibility in the two cell types, there were significant but very modest differences in mean expression levels. At promoters that were hyperaccessible in DKO cells, we observed substantial and significantly higher levels of expression in DKO cells relative to HCT116, with expression differences increasing as accessibility increased (Fig. 3F). These are in accordance with previous findings (Kelly et al. 2012), further validating the utility of mATAC-seq and demonstrating the concordance between the extent of chromatin accessibility at promoters and promoter activity.

Our analyses so far have separately examined methylation and chromatin accessibility results from mATAC-seq. We next combined methylation and accessibility data to take advantage of added value of the combined results afforded by mATAC-seq. We first performed $k$-means clustering of DNA methylation levels at THS sites in HCT116 and DKO cells. DNA methylation at mATAC-seq peaks in HCT116 cells formed five distinct clusters (Fig. 4A). In Cluster 1 , accessible peaks, and the $1 \mathrm{~kb}$ intervals flanking the peaks, were hypermethylated in HCT116 relative to DKO cells, with the flanks exhibiting more hypermethylation. Clusters 2 


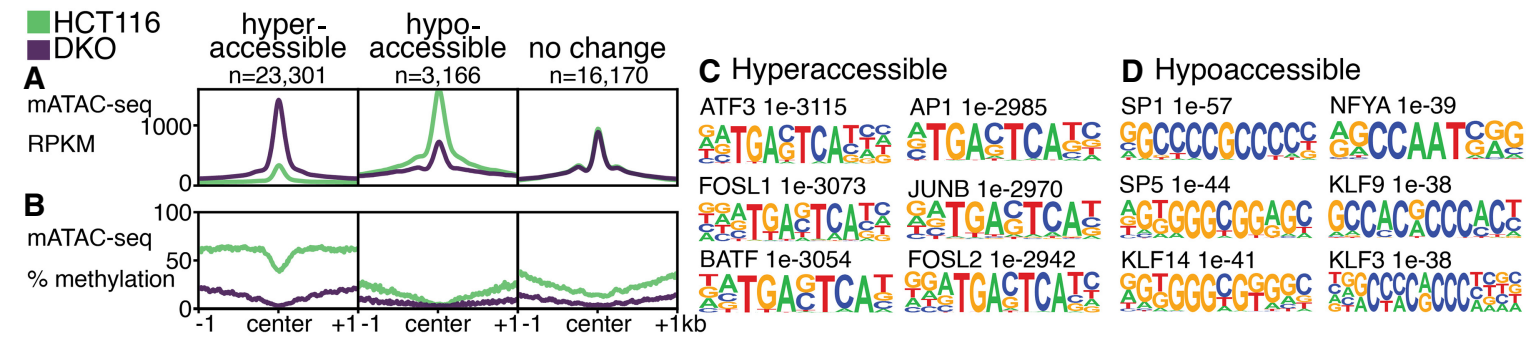

E Differential methylation vs accessibility at promoters

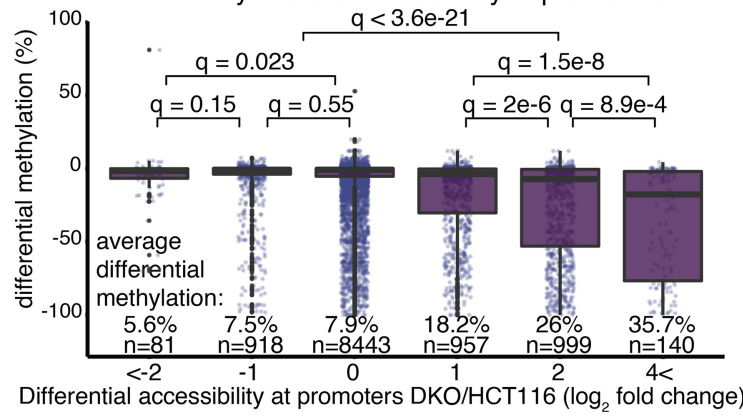

F mRNA expression vs differential accessibility at promoters

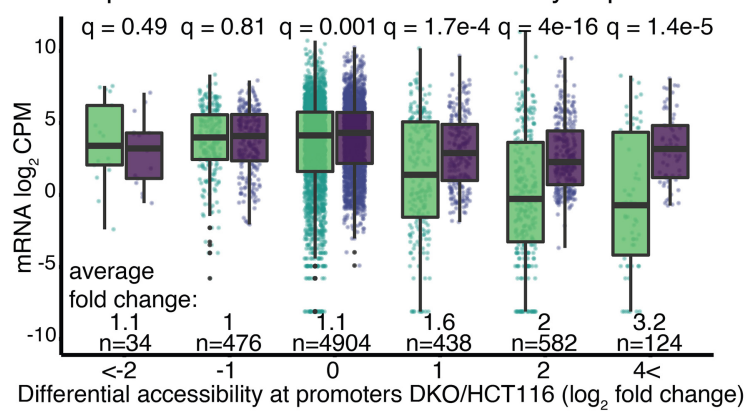

Figure 3. Accessibility and methylation at peaks. $(A, B)$ Significantly changed mATAC-seq hyperaccessible $\left(\log _{2}\right.$ fold change $>1, q<0.01, n=23,310$ peaks), hypoaccessible $\left(\log _{2}\right.$ fold change $<-1, q<0.01, n=3166$ peaks), and unchanged peaks $\left(\mid \log _{2}\right.$ fold change $\mid<1, q>0.8 n=16,170$ ). Motifs enriched in hyperaccessible $(C)$ and hypoaccessible $(D)$ sites compared to unchanged sites. $(E)$ DNA methylation changes at promoters binned by accessibility, reported as the change in methylation ratio of DKO cells relative to HCT116 (DKO/HCT116). (F) mRNA expression changes in DKO cells relative to $\mathrm{HCT116}$, reported as $\log _{2}$ CPM, at genes binned by differential accessibility of their promoters as in $E$. Q-values are for Wilcoxon tests with BenjaminiHochberg correction (Benjamini and Hochberg 1995).

and 3 were hypomethylated at peak centers in both cells; the clusters were respectively hypermethylated in HCT116 cells in one or the other of the two intervals flanking the peaks. Cluster 4 was hypermethylated over the peaks only in HCT116 cells and hypomethylated in the peak and flanks in DKO cells. Cluster 5 was hypomethylated in the peaks and flanks of both cell types (Fig. 4A,C,D).

When we assessed mRNA expression from promoters within the five clusters, differences between DKO and HCT116 emerged that varied according to cluster. Promoters in DKO cells from Clusters 1 and 4 were significantly more active than the corresponding promoters from the same clusters in HCT116 cells, with respective increases in mRNA of 2.5- and 3.2-fold (Fig. 4B). Clusters 2 and 3 exhibited a modest change of 1.3 -fold between the cell types. Cluster 5, which was both hypomethylated and hyperaccessible in both cell types, showed no difference in expression.

Besides the differences in DNA methylation and expression, the clusters have additional distinguishing features. There are more promoters, CpG islands, and exons in Cluster 1 compared to Cluster 4; and more intronic and distal intergenic elements in Cluster 4 compared to Cluster 1 (Supplemental Fig. S3A,B). One feature is the broad domain of H2A.Z in Cluster 1 that accompanied the loss of DNA methylation in DKO cells (Fig. 4E). This finding is consistent with reports that DNA modification and H2A.Z are mutually antagonistic (Zilberman et al. 2008). In Cluster 4, where hypermethylation in HCT116 cells is largely confined to the mATAC-seq peak, there was also an increase in H2A.Z in DKO cells, with the increase being more modest and confined to a narrower portion of the $2 \mathrm{~kb}$ window displayed. Additional histone modifications associated with active chromatin (H3K4me1, H3K4me3, H3K27ac) were elevated in DKO cells near Cluster 1
mATAC-seq peaks, but these effects were limited or absent in Cluster 4 (Fig. 4E). Like H2A.Z, H3K27me3 was increased in DKO cells at Cluster 1, with the effects also being more modest at Cluster 4 (Fig. 4F). This is also consistent with antagonism reported between H3K27me3 and DNA methylation (Lindroth et al. 2008). In contrast to these histone modifications and variants, H3K9me3 at mATAC peaks was largely unaffected by DNMT loss. Cluster 5 shows no DNA methylation changes between the two conditions, and there were few to no changes in deposition of histone modifications and variants.

Motifs for TFs and CTCF (Fig. 4G) binding also varied by cluster. Cluster 1 is enriched for motifs recognized by DNA methylplus TFs such as CTCFL, MYC, and BHLHE40; ZFX and ZNF711 contain similar motifs to ZNF704, a methyl-minus transcription factor (Fig. 4H). Of the top five TFs enriched in Cluster 4, three are MEF-family TFs, followed by ARNT, which was previously suggested to be methyl-sensitive (Fig. 4I; Lay et al. 2015). ARNT motifs share substantial sequence identity with BHLHE40, a methyl-minus TF.

\section{Discussion}

ATAC-seq identifies nucleosome-depleted regions of the genome, which are relevant for gene regulation within cells. By including bisulfite treatment in the workflow, mATAC-seq targets DNA methylation profiling to open chromatin sites that are enriched for active regulatory regions of the genome. Accordingly, mATAC-seq queries the functional methylome of cells, using relatively few reads compared to WGBS. This is in contrast to other assays for DNA methylation that query the entire genome or other domains that may not be regulatory.

\section{Genome Research}

www.genome.org 


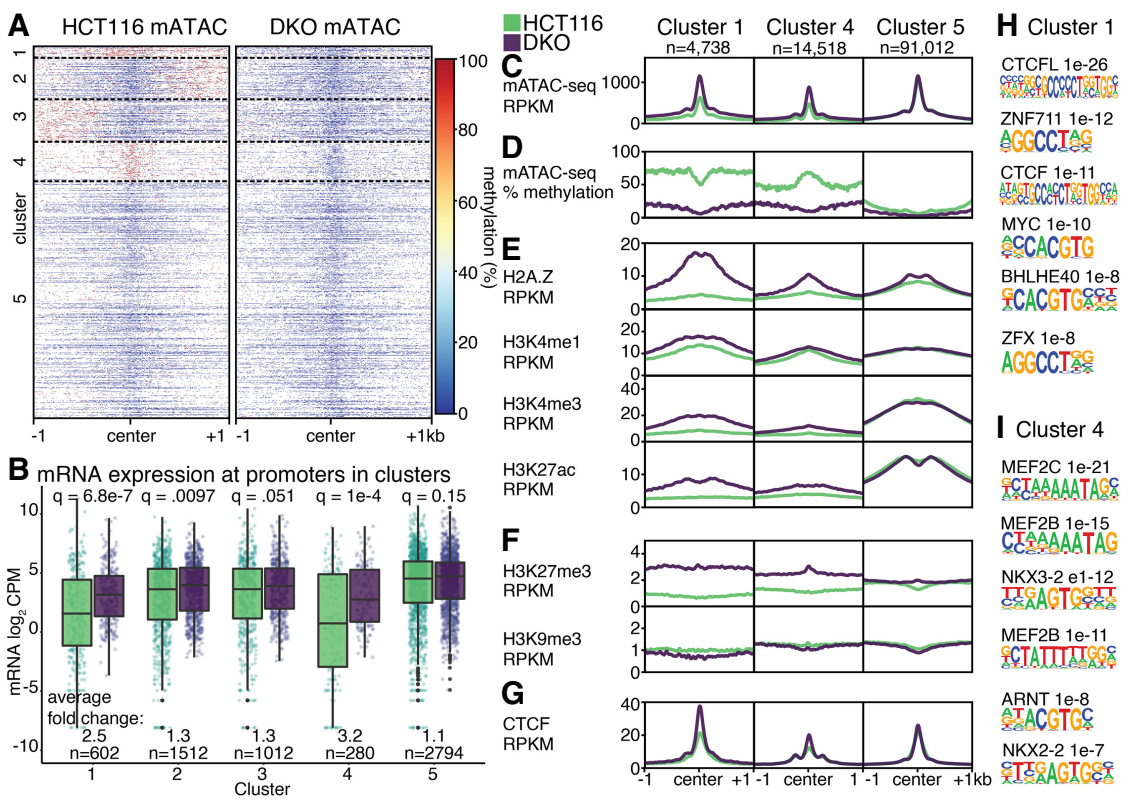

Figure 4. Combined accessibility and methylation analysis. $(A)$ DNA methylation at mATAC-seq peaks from HCT116 and DKO cells form five distinct clusters by DNA methylation. (B) mRNA expression in log 2 CPM at identified clusters. Features in Clusters 1, 4, and 5 are depicted according to accessibility (C), DNA methylation $(D)$, activating histone modifications $(E)$, silencing histone modifications $(F)$, and CTCF $(G)$. Motifs enriched in Cluster $1(H)$ and Cluster $4(I)$, compared to Cluster 5. Q-values are for Wilcoxon tests with Benjamini-Hochberg correction.

By applying mATAC-seq to the well-characterized HCT116 cell line, and its DNA methylation-deficient DKO derivative, we demonstrated that mATAC-seq detects DNA methylation patterns that agree with both previously described WGBS results and with our Omni-ATAC-seq results. These tests validated the fidelity and compatibility of combining tagmentation and bisulfite treatment steps in the mATAC-seq workflow. DKO cells had many hyperaccessible sites relative to parental HCT116 cells, and these sites exhibited loss of methylation. These same regions were also enriched for methyl-minus TF binding sites, which interact more strongly with DNA when the sites are in an unmethylated context. This highlights the instructive role of TF binding for nucleosome occupancy in the genome. Specifically, our data indicate that when DNA is unmethylated, it facilitates the recruitment of methyl-minus TFs, and that these in turn enable chromatin to assume an open state. Our data also revealed that hyperaccessible and hypomethylated domains in DKO cells were enriched for the histone variant H2A.Z, implicating this factor in limiting DNA methylation, and nucleosome density at sequences where it is recruited. In contrast, regions that displayed no change in methylation showed little change in accessibility. We did not observe a depletion of H3K9me3 at sites with increased accessibility and decreased DNA methylation, confirming statements in previous studies (Blattler et al. 2014). Such findings were made possible by the combination of DNA methylation and open chromatin status provided by mATAC-seq.

We envisage that mATAC-seq could be applied to many other systems. For example, HCT116 derivatives carrying single DNMT knockouts (Rhee et al. 2000; Rhee et al. 2002) would enable us to identify regulatory elements the different DNMTs individually target for methylation and their respective influences on nucleosome placement. The various DNMTs have been shown to regulate DNA methylation states by independent as well as coop- erative mechanisms (Liang et al. 2002). Repetitive elements are common targets of the DNMTs, and the resulting DNA methylation contributes to their silencing. However, silencing can occur when DNMT activities are impaired, indicating that compensating mechanisms can silence transposons, likely involving H3K9 methylation, and possibly other chromatin modifications (Horard et al. 2009; Karimi et al. 2011; Walter et al. 2016; Jordà et al. 2017). Querying the specificities of the DNMTs and their influences on chromatin accessibility at repetitive elements using mATAC-seq can elaborate mechanisms underlying repeat regulation.

Additionally, by using HCT116 DKO cells, we studied the effects of DNA methylation depletion that arose by passive mechanisms owing to a lack of DNA methylation maintenance. Active demethylation by TET dioxygenases, and AICDA/APOBEC deaminases occurring during differentiation and response to stimuli, is a distinct process. Applying mATAC-seq to stem cell differentiation, including under conditions in which these active demethylation mechanisms are altered, can reveal both the combinatorial changes in accessibility and DNA methylation and the effects active DNA methylation mechanisms have on chromatin state during differentiation.

Pioneer transcription factors have the unique property of binding chromatin that is generally inaccessible to other transcription factors (Zaret and Mango 2016). Application of mATAC-seq to systems in which pioneer factor functions are altered can reveal the influences these factors have on both chromatin accessibility and methylation state of the underlying DNA.

Our protocol for mATAC-seq can potentially be integrated with existing methods for combinatorial detection of other DNA modifications including 5-hydroxymethylcytosine ( $\mathrm{Yu}$ et al. 2012; Booth et al. 2013), 5-formylcytosine (Song et al. 2013), and 5-carboxylcytosine ( $\mathrm{Wu}$ et al. 2016). ChIPmentation uses Tn5 tagmentation in a chromatin immunoprecipitation workflow (Schmidl et al. 2015). This too could be implemented, using steps we developed for mATAC-seq, to identify locations of DNA-bound proteins and the underlying DNA modification states in a combinatorial detection strategy similar to other methyl-ChIP strategies (Brinkman et al. 2012; Statham et al. 2012). Combinatorial indexing as a low-cost strategy to query single cells can be used to enable the extension of mATAC-seq to a single-cell format; specifically, methods such as single-cell combinatorial indexing assay for transposase accessible chromatin using sequencing (sci-ATAC-seq) (Cusanovich et al. 2015), and for methylation analysis (sci-MET) (Mulqueen et al. 2018). Some alterations are necessary to adapt our mATAC-seq protocol for single-cell sequencing, including extending the indexed adapter set to use methylated sci-ATAC-seq adapters during tagmentation, followed by split pooling, methylated end repair, bisulfite conversion in a 96-well format, and PCR. The challenge to this approach is the depletion of reads caused by the destructiveness of bisulfite conversion and the limited sequence complexity in bisulfite converted reads. 


\section{Methods}

\section{Cell culture}

Cultured cells (\#28 HCT116 Parental and \#343 DKO) were procured from the Genetic Resources Core Facility at Johns Hopkins School of Medicine and cultured in McCoy's Modified 5A Medium containing 10\% heat-inactivated FBS and $1 \times$ Penn/Strep (Gibco \#15140122). Cells for each experiment were grown apart for at least two passages before library preparation.

\section{Genotyping}

DNA from each cell line was extracted using EZ-10 Spin Columns (Bio Basic \#BS427) following the manufacturer's protocol. Genotyping PCR was performed on 50 ng genomic DNA using oligos from Supplemental Table S2 from Das and Chadwick (2016) for 40 cycles using GoTaq (Promega \#M3001) $\left(2 \mathrm{~min}\right.$ at $94^{\circ} \mathrm{C}$; 40 cycles of the following: $30 \mathrm{sec}$ at $94^{\circ} \mathrm{C}, 30 \mathrm{sec}$ at $60^{\circ} \mathrm{C}, 30 \mathrm{sec}$ at $72^{\circ} \mathrm{C}$; then $5 \mathrm{~min}$ at $72^{\circ} \mathrm{C}$ ) and run on a $2 \%$ agarose gel. Cells were confirmed to be Mycoplasma-free and HeLa-free via PCR (Rahbari et al. 2009; Young et al. 2010) on 50 ng genomic DNA and cell culture media (Supplemental Fig. S4).

\section{Tn5 transposase}

Tn5 was produced exactly as described (Picelli et al. 2014) with no modifications. Enzyme was stored at $-80^{\circ} \mathrm{C}$. For Omni-ATAC-seq, Tn5 transposase was assembled as in Adey and Shendure (2012). Oligonucleotides in Supplemental Table S3 were annealed by combining ME-A or ME-B oligos to Tn5MErev and incubating for $2 \mathrm{~min}$ at $94^{\circ} \mathrm{C}$ followed by a $0.1^{\circ} \mathrm{C} / \mathrm{sec}$ ramp to $25^{\circ} \mathrm{C}$. Twenty-five microliters of $20 \mu \mathrm{M}$ annealed Tn5MEDS-A and $25 \mu \mathrm{L}$ of $20 \mu \mathrm{M}$ annealed Tn5MEDS-B were combined with $50 \mu \mathrm{L}$ glycerol. Annealed oligonucleotides in $50 \%$ glycerol were mixed with an equal volume of Tn5 transposase concentrated to A280 $=3.0$. For methylATACseq, Tn5 transposase was assembled identically to Omni-ATACseq using pre-annealed Tn5ME-A_5mC and Tn5MEB_5mC oligonucleotides from Supplemental Table S3.

\section{Omni-ATAC-seq}

Cells were trypsinized and subsequently inactivated in cell culture media. Following inactivation, cells were pelleted and resuspended in cold PBS (without $\mathrm{Ca++}$ and $\mathrm{Mg}++$ ). Cells were stained with Trypan Blue and counted on a hemocytometer. Lysis and tagmentation were performed exactly as described (Corces et al. 2017) with modifications to inactivation and size selection. Briefly, 100,000 HCT116 Parental and DKO cells were lysed on ice for 3 min in $50 \mu \mathrm{L}$ ice-cold Lysis Buffer $(10 \mathrm{mM}$ Tris [pH 7.4], $10 \mathrm{mM}$ $\mathrm{NaCl}, 3 \mathrm{mM} \mathrm{MgCl}_{2}, 0.1 \% \mathrm{NP}-40,0.1 \%$ Tween 20, $0.01 \%$ Digitonin in DEPC $\mathrm{H}_{2} \mathrm{O}$ ), resuspended in $1 \mathrm{~mL}$ ice-cold RBSWash (10 mM Tris [pH 7.4], $10 \mathrm{mM} \mathrm{NaCl}, 3 \mathrm{mM} \mathrm{MgCl}_{2}, 0.1 \%$ Tween 20), and pelleted at $4^{\circ} \mathrm{C}$ at $500 \mathrm{~g}$ for $10 \mathrm{~min}$. Tagmentation was performed in $1 \times$ Tagmentation Buffer $(10 \mathrm{mM}$ Tris [pH 7.4], $5 \mathrm{mM} \mathrm{MgCl}_{2}, 10 \%$ DMF, 33\% PBS, $0.1 \%$ Tween 20, $0.01 \%$ Digitonin) using $1 \mu \mathrm{L}$ of Tn5 transposase assembled using pre-annealed Tn5MEDS-A and Tn5MEDS-B for $30 \mathrm{~min}$ at $37^{\circ} \mathrm{C}$. Tagmentation was inactivated with the addition of 5 volumes SDS Lysis Buffer (100 mM Tris [pH 7.4], $50 \mathrm{mM} \mathrm{NaCl}, 10 \mathrm{mM}$ EDTA, $0.5 \%$ SDS in $\mathrm{H}_{2} \mathrm{O}$ ) and $100 \mu \mathrm{g}$ Proteinase $\mathrm{K}$ (Invitrogen \#25530049) for $30 \mathrm{~min}$ at $55^{\circ} \mathrm{C}$ followed by Isopropanol precipitation using GlycoBlue (Invitrogen \#AM9516) as a carrier. DNA was size selected using Ampure XP beads (Beckman Coulter \# A63880) using $0.5 \times$ volume to remove large fragments followed by a $1.8 \times$ final volume according to the manufacturer's instructions. PCR was performed by using Q5 DNA polymerase (NEB \#M0491S) with 1×
GC buffer $\left(5 \mathrm{~min}\right.$ at $72^{\circ} \mathrm{C}, 30 \mathrm{sec}$ at $98^{\circ} \mathrm{C} ; 11$ cycles of the following: $10 \mathrm{sec}$ at $98^{\circ} \mathrm{C}, 30 \mathrm{sec}$ at $65^{\circ} \mathrm{C}, 30 \mathrm{sec}$ at $72^{\circ} \mathrm{C}$; and then $5 \mathrm{~min}$ at $72^{\circ} \mathrm{C}$ ) followed by a final cleanup using $1.8 \times$ volume of Ampure $\mathrm{XP}$ beads according to the manufacturer's instructions.

\section{methyl-ATAC-seq}

Cell lysis was performed identically to Omni-ATAC-seq. Tagmentation was performed on 250,000 HCT116 Parental and DKO cells using $7 \mu \mathrm{L}$ of Tn5 transposase assembled using pre-annealed Tn5ME-A_mC and Tn5ME-B_mC (Supplemental Table S3) for $30 \mathrm{~min}$ at $37^{\circ} \mathrm{C}$ following the addition of $0.01 \mathrm{ng}$ unmethylated Lambda DNA (Promega \#D1521). We recommend performing a titration of Tn5 transposase to nuclei input to assay minimum amounts required as in Supplemental Figure S5. Inactivation and size selection were performed identically to our modified Omni-ATAC-seq protocol. Tagmented DNA was end-repaired for $30 \mathrm{~min}$ at $37^{\circ} \mathrm{C}$ (5 units Klenow Exo- [NEB \#M2012S], 1× NEB Buffer 2, and $0.5 \mathrm{mM} /$ each dATP, dGTP, dTTP, and 5-mdCTP [NEB \#N0365S]) similar to T-WGBS (Lu et al. 2015) and X-WGBS (Suzuki et al. 2018). End repair was cleaned using 1.8× volume of Ampure XP beads according to the manufacturer's instructions. Ten percent of the product was kept for quality control PCR (Supplemental Fig. S6A). Bisulfite conversion was performed using EZ DNA Methylation-Lightning (Zymo \#D5030T) following the manufacturer's protocol. PCR was immediately performed using PfuTurbo Cx (Agilent \#600410) $\left(2 \mathrm{~min}\right.$ at $94^{\circ} \mathrm{C} ; 13$ cycles of the following: $10 \mathrm{sec}$ at $98^{\circ} \mathrm{C}, 30 \mathrm{sec}$ at $65^{\circ} \mathrm{C}, 30 \mathrm{sec}$ at $72^{\circ} \mathrm{C}$; then $5 \mathrm{~min}$ at $72^{\circ} \mathrm{C}$ ) (Supplemental Fig. S6B) followed by a final cleanup using $1.8 \times$ volume of Ampure XP beads according to the manufacturer's instructions.

\section{Data analysis}

Libraries were quantified using the Qubit dsDNA HS Assay Kit (Thermo Fisher Scientific \#Q32854). High-throughput sequencing was performed by the Cornell University Genomics Facility on the Illumina NextSeq 500 with single-end 75 -bp reads. Trimming for mATAC-seq and Omni-ATAC-seq was performed using fastp (Chen et al. 2018) -q 20 -1 20 -a CTGTCTCTTATACACATCT. Trimming for ChIP-seq, RNA-seq, and WGBS data was performed using fastp -q 20 -1 20 -a AGATCGGAAGAGCACACGTCTGAA CTCCAGTCAC.

\section{Alignment to hg19}

In this study we used GRCh37 instead of GRCh38 to match previous studies using similar cells and methods. These results would not be affected by this change, because we do not study centromeric sequences and predominantly discuss changes at promoters.

\section{ChIP-seq hg19}

Trimmed FASTQ files were aligned using BWA-MEM (Li and Durbin 2010) to hg19. Reads were deduplicated using Picard (http:// broadinstitute.github.io/picard/) MarkDuplicates. HCT116 and DKO ChIP-seq data for H2A.Z, H3K4me3, H3K4me1, H3K27ac, H3K27me3, H3K9me3, and H3K36me3 data (Lay et al. 2015) were downloaded from NCBI Gene Expression Omnibus (GEO) database accession GSE58638. HCT116 and DKO ChIP-seq data for CTCF (Maurano et al. 2015) were downloaded from NCBI GEO database accession GSE50610.

\section{RNA-seq hg19}

Pair-end trimmed FASTQ files were aligned using HISAT2 (Kim et al. 2015) to hg19. HCT116 and DKO RNA-seq data (Blattler

\section{Genome Research}

www.genome.org 
et al. 2014) were downloaded from NCBI GEO database accessions GSE52429 and GSE60106, respectively.

\section{Omni-ATAC and mATAC-seq}

Trimmed FASTQ files were aligned using Bismark (Krueger and Andrews 2011) v0.19.0 to hg19 using the following settings: --score_min L,0,-0.6. Bisulfite reads to be used for methylKit (Akalin et al. 2012) were filtered for nonconversion using Bismark's filter_non_conversion and deduplicated using deduplicate_bismark. Methylation was extracted using Bismark's methylation extractor --gzip --bedgraph --counts --ignore 9 --ignore_3prime 9. Reads used for peak calling and ATAC-seq visualization were deduplicated using deduplicate_ bismark without filtering for nonconversion. Conversion rate (Supplemental Table S1) was measured by aligning to the lambda genome (GenBank: J02459.1) and filtering as above; percent conversion rate was calculated as [1-(Total methylated Cs in all contexts)/ (Total number of Cs analyzed) $] \times 100$.

\section{WGBS hg19}

Trimmed FASTQ files were aligned using Bismark v0.19.0 to hg19 using the following settings: --score_min L,0,-0.6. Bisulfite reads to be used for methylKit were filtered for nonconversion using Bismark's filter_non_conversion and deduplicated using deduplicate_bismark. methylation was extracted using Bismark's methylation extractor --gzip --bedgraph --counts. HCT116 and DKO WGBS data (Blattler et al. 2014) were downloaded from NCBI GEO database accession GSE60106.

\section{Methylation}

Differential methylation was quantified using methylKit at merged HCT116 and DKO mATAC-seq peaks extended to 1-kb tiles covering at least three CpGs. Promoters were defined as being within $1 \mathrm{~kb}$ of a TSS using Genomation (Akalin et al. 2015).

\section{Peak calling}

ATAC-seq peaks were called using HOMER (Heinz et al. 2010) findPeaks localSize 50000 -size 150 -minDist 50 -fragLength 0 -style dnase. ChIP-seq peaks were called using HOMER findPeaks -style histone. CTCF ChIP-seq peaks were called using HOMER findPeaks -style factor. Reads were assigned to peaks merged from HCT116 and DKO cells using featureCounts (Liao et al. 2013) on reads filtered for a minimum $\log _{2}$ CPM of 0.5 in at least two samples. Differential accessibility was called using DESeq2 (Love et al. 2014) lfcShrink. Hyper- and hypoaccessible peaks were defined as having a $\left|\log _{2} \mathrm{FC}\right|>1$ with an adjusted $P$-value $<0.01$ in DKO compared to HCT116 parental cells. Promoters were defined as being within $1 \mathrm{~kb}$ of a TSS using Genomation. FRiP scores in Supplemental Table S1 and sample correlation in Supplemental Figure S2 were quantified using DiffBind (http:// bioconductor.org/packages/release/bioc/html/DiffBind.html) on libraries downsampled to $5 \mathrm{M}$ reads using Picard DownsampleSam using peaks called by HOMER. Peak overlaps for Figure 1A and Supplemental Figure S2E were generated using ChIPpeakAnno (Zhu et al. 2010). Feature overlaps for Figure 1B and Supplemental S3A were generated using ChIPseeker (Yu et al. 2015). Motif enriched in changed peaks were called using HOMER findMotifsGenome to the hg19 genome using unchanged peaks as background.

\section{RNA-seq quantification}

Unstranded hg19-aligned reads were assigned to hg19 genes using featureCounts inbuilt reference using default settings. Differential expression was quantified using DESeq2 lfcShrink on reads filtered for a minimum CPM of 0.5 in at least two samples.

\section{Genome Browser visualizations}

ATAC-seq and mATAC-seq bigWig files were made using deepTools (Ramírez et al. 2016) bamCoverage --binSize 1 --normalizeUsing RPKM --ignoreForNormalization chrM --scaleFactor $\mathrm{N}$ and viewed on the UCSC Genome Browser. ChIP-seq bigWigs were made using deepTools bamCoverage --binSize 10 --normalizeUsing RPKM --ignoreForNormalization chrM --scaleFactor N. Scale factor was determined by coverage of peaks called by HOMER shared between HCT116 and DKO via bedops --intersect where $N=(\%$ reads in shared peaks in HCT116)/(\% reads in shared peaks in DKO) when $N>1.1$. Scaling was applied to the following samples: DKO_mATAC 1.877,DKO_H3K27ac_R2 1.48, H3K4me3_R2=1.47.

Gene body heatmaps were produced using deepTools plotheatmap --beforeRegionStartLength 2000 --regionBodyLength 3000 --afterRegionStartLength 2000 to Ensembl hg19 APPRIS PRINCIPAL:1 flagged transcripts (Rodriguez et al. 2013). Heatmaps for differential peaks were centered on peaks called by HOMER. Peaks from HCT116 and DKO were combined using BEDOPS for clustering of DNA methylation at THS sites. Clustering was performed using deepTools plotheatmap --kmeans 5 , the output and order of which was used for all subsequent heatmaps.

\section{Data access}

All raw and processed sequencing data generated in this study have been submitted to the NCBI Gene Expression Omnibus (GEO; http://www.ncbi.nlm.nih.gov/geo/) under accession number GSE126215.

\section{Acknowledgments}

We thank Jennifer D. Mosher, Ann E. Tate, Jeff C. Mattison, and Peter A. Schweitzer from the Cornell University Biotechnology Resource Center (BRC) for genomic sequencing; Bert Vogelstein for HCT116 cells and derivatives; Önder Kartal and Erin Chu for proofreading and helpful comments; and the Cornell University, College of Veterinary Medicine and the National Institutes of Health for funding (R01 HG006850, R01 GM105243, and T32 GM007617).

\section{References}

Adey A, Shendure J. 2012. Ultra-low-input, tagmentation-based whole-genome bisulfite sequencing. Genome Res 22: 1139-1143. doi:10.1101/ gr.136242.111

Akalin A, Kormaksson M, Li S, Garrett-Bakelman FE, Figueroa ME, Melnick A, Mason CE. 2012. methylKit: a comprehensive R package for the analysis of genome-wide DNA methylation profiles. Genome Biol 13: R87. doi:10.1186/gb-2012-13-10-r87

Akalin A, Franke V, Vlahoviček K, Mason CE, Schübeler D. 2015. Genomation: a toolkit to summarize, annotate and visualize genomic intervals. Bioinformatics 31: 1127-1129. doi:10.1093/bioinformatics/ btu775

Benjamini Y, Hochberg Y. 1995. Controlling the false discovery rate: a practical and powerful approach to multiple testing. J R Stat Soc Ser B: Stat Methodol 57: 289-300. doi:10.1111/j.2517-6161.1995.tb02031.x

Bernstein BE, Mikkelsen TS, Xie X, Kamal M, Huebert DJ, Cuff J, Fry B, Meissner A, Wernig M, Plath K, et al. 2006. A bivalent chromatin structure marks key developmental genes in embryonic stem cells. Cell 125: 315-326. doi:10.1016/j.cell.2006.02.041 
Blattler A, Yao L, Witt H, Guo Y, Nicolet CM, Berman BP, Farnham PJ. 2014 Global loss of DNA methylation uncovers intronic enhancers in genes showing expression changes. Genome Biol 15: 469. doi:10.1186/ s13059-014-0469-0

Booth MJ, Ost TW, Beraldi D, Bell NM, Branco MR, Reik W, Balasubramanian S. 2013. Oxidative bisulfite sequencing of 5-methylcytosine and 5-hydroxymethylcytosine. Nat Protoc 8: 1841-1851. doi:10.1038/ nprot.2013.115

Boyle P, Clement K, Gu H, Smith ZD, Ziller M, Fostel JL, Holmes L, Meldrim J, Kelley F, Gnirke A, et al. 2012. Gel-free multiplexed reduced representation bisulfite sequencing for large-scale DNA methylation profiling. Genome Biol 13: R92. doi:10.1186/gb-2012-13-10-r92

Brinkman AB, Gu H, Bartels SJ, Zhang Y, Matarese F, Simmer F, Marks $\mathrm{H}_{\text {, }}$ Bock C, Gnirke A, Meissner A, et al. 2012. Sequential ChIP-bisulfite sequencing enables direct genome-scale investigation of chromatin and DNA methylation cross-talk. Genome Res 22: 1128-1138. doi:10.1101/ gr.133728.111

Buenrostro JD, Giresi PG, Zaba LC, Chang HY, Greenleaf WJ. 2013. Transposition of native chromatin for fast and sensitive epigenomic profiling of open chromatin, DNA-binding proteins and nucleosome position. Nat Methods 10: 1213-1218. doi:10.1038/nmeth.2688

Chatterjee A, Rodger EJ, Stockwell PA, Weeks RJ, Morison IM. 2012. Technical considerations for reduced representation bisulfite sequencing with multiplexed libraries. J Biomed Biotechnol 2012: 741542. doi:10.1155/2012/741542

Chen S, Zhou Y, Chen Y, Gu J. 2018. fastp: an ultra-fast all-in-one FASTQ preprocessor. Bioinformatics 34: i884-i890. doi:10.1093/bioinfor matics/bty560

Corces MR, Buenrostro JD, Wu B, Greenside PG, Chan SM, Koenig JL, Snyder MP, Pritchard JK, Kundaje A, Greenleaf WJ, et al. 2016. Lineage-specific and single-cell chromatin accessibility charts human hematopoiesis and leukemia evolution. Nat Genet 48: 1193-1203. doi:10.1038/ng.3646

Corces MR, Trevino AE, Hamilton EG, Greenside PG, Sinnott-Armstrong NA, Vesuna S, Satpathy AT, Rubin AJ, Montine KS, Wu B, et al. 2017. An improved ATAC-seq protocol reduces background and enables interrogation of frozen tissues. Nat Methods 14: 959-962. doi:10.1038/ nmeth.4396

Cusanovich DA, Daza R, Adey A, Pliner H, Christiansen L, Gunderson KL, Steemers FJ, Trapnell C, Shendure J. 2015. Multiplex single-cell profiling of chromatin accessibility by combinatorial cellular indexing. Science 348: 910-914. doi:10.1126/science.aab1601

Das S, Chadwick BP. 2016. Influence of repressive histone and DNA methylation upon D4Z4 transcription in non-myogenic cells. PLoS One 11: e0160022. doi:10.1371/journal.pone.0160022

Garrett-Bakelman FE, Sheridan CK, Kacmarczyk TJ, Ishii J, Betel D, Alonso A, Mason CE, Figueroa ME, Melnick AM. 2015. Enhanced reduced representation bisulfite sequencing for assessment of DNA methylation at base pair resolution. J Vis Exp 96: e52246. doi:10.3791/52246

Gaulton KJ, Nammo T, Pasquali L, Simon JM, Giresi PG, Fogarty MP, Panhuis TM, Mieczkowski P, Secchi A, Bosco D, et al. 2010. A map of open chromatin in human pancreatic islets. Nat Genet 42: 255-259. doi:10.1038/ng.530

Heintzman ND, Hon GC, Hawkins RD, Kheradpour P, Stark A, Harp LF, Ye Z Lee LK, Stuart RK, Ching CW, et al. 2009. Histone modifications at human enhancers reflect global cell-type-specific gene expression. Nature 459: 108-112. doi: 10.1038 /nature07829

Heinz S, Benner C, Spann N, Bertolino E, Lin YC, Laslo P, Cheng JX, Murre C, Singh H, Glass CK. 2010. Simple combinations of lineage-determining transcription factors prime cis-regulatory elements required for macrophage and B cell identities. Mol Cell 38: 576-589. doi:10.1016/j molcel.2010.05.004

Horard B, Eymery A, Fourel G, Vassetzky N, Puechberty J, Roizes G, Lebrigand K, Barbry P, Laugraud A, Gautier C, et al. 2009. Global analysis of DNA methylation and transcription of human repetitive sequences. Epigenetics 4: 339-350. doi:10.4161/epi.4.5.9284

Jordà M, Díez-Villanueva A, Mallona I, Martín B, Lois S, Barrera V, Esteller M, Vavouri T, Peinado MA. 2017. The epigenetic landscape of Alu repeats delineates the structural and functional genomic architecture of colon cancer cells. Genome Res 27: 118-132. doi:10.1101/gr.207522.116

Karimi MM, Goyal P, Maksakova IA, Bilenky M, Leung D, Tang JX, Shinkai Y, Mager DL, Jones S, Hirst M, et al. 2011. DNA methylation and SETDB1/H3K9me3 regulate predominantly distinct sets of genes, retroelements, and chimeric transcripts in mESCs. Cell Stem Cell 8: 676-687. doi:10.1016/j.stem.2011.04.004

Kelly TK, Liu Y, Lay FD, Liang G, Berman BP, Jones PA. 2012. Genome-wide mapping of nucleosome positioning and DNA methylation within individual DNA molecules. Genome Res 22: 2497-2506. doi:10.1101/gr .143008 .112
Kim D, Langmead B, Salzberg SL. 2015. HISAT: a fast spliced aligner with low memory requirements. Nat Methods 12: 357-360. doi:10.1038/nmeth 3317

Krueger F, Andrews SR. 2011. Bismark: a flexible aligner and methylation caller for Bisulfite-Seq applications. Bioinformatics 27: 1571-1572. doi:10.1093/bioinformatics/btr167

Lay FD, Liu Y, Kelly TK, Witt H, Farnham PJ, Jones PA, Berman BP. 2015. The role of DNA methylation in directing the functional organization of the cancer epigenome. Genome Res 25: 467-477. doi:10.1101/gr.183368 114

Li H, Durbin R. 2010. Fast and accurate long-read alignment with BurrowsWheeler transform. Bioinformatics 26: 589-595. doi:10.1093/bioinfor matics/btp698

Liang G, Chan MF, Tomigahara Y, Tsai YC, Gonzales FA, Li E, Laird PW, Jones PA. 2002. Cooperativity between DNA methyltransferases in the maintenance methylation of repetitive elements. Mol Cell Biol 22: 480-491. doi:10.1128/MCB.22.2.480-491.2002

Liao Y, Smyth GK, Shi W. 2013. The Subread aligner: fast, accurate and scalable read mapping by seed-and-vote. Nucleic Acids Res 41: e108. doi:10 $.1093 /$ nar/gkt214

Lindroth AM, Park YJ, McLean CM, Dokshin GA, Persson JM, Herman H, Pasini D, Miro X, Donohoe ME, Lee JT, et al. 2008. Antagonism between DNA and H3K27 methylation at the imprinted Rasgrf1 locus. PLoS Genet 4: e1000145. doi:10.1371/journal.pgen.1000145

Love MI, Huber W, Anders S. 2014. Moderated estimation of fold change and dispersion for RNA-seq data with DESeq2. Genome Biol 15: 550 . doi:10.1186/s13059-014-0550-8

Lu H, Yuan Z, Tan T, Wang J, Zhang J, Luo HJ, Xia Y, Ji W, Gao F. 2015. Improved tagmentation-based whole-genome bisulfite sequencing for input DNA from less than 100 mammalian cells. Epigenomics 7: 47-56. doi:10.2217/epi.14.76

Maurano MT, Wang H, John S, Shafer A, Canfield T, Lee K, Stamatoyannopoulos JA. 2015. Role of DNA methylation in modulating transcription factor occupancy. Cell Rep 12: 1184-1195. doi:10.1016/ .celrep.2015.07.024

Meissner A, Gnirke A, Bell GW, Ramsahoye B, Lander ES, Jaenisch R. 2005. Reduced representation bisulfite sequencing for comparative high-resolution DNA methylation analysis. Nucleic Acids Res 33: 5868-5877. doi:10.1093/nar/gki901

Mulqueen RM, Pokholok D, Norberg SJ, Torkenczy KA, Fields AJ, Sun D, Sinnamon JR, Shendure J, Trapnell C, O'Roak BJ, et al. 2018. Highly scalable generation of DNA methylation profiles in single cells. Nat Biotechnol 36: 428-431. doi:10.1038/nbt.4112

Picelli S, Björklund ÅK, Reinius B, Sagasser S, Winberg G, Sandberg R. 2014. Tn5 transposase and tagmentation procedures for massively scaled sequencing projects. Genome Res 24: 2033-2040. doi:10.1101/gr.177881 .114

Rahbari R, Sheahan T, Modes V, Collier P, Macfarlane C, Badge RM. 2009. A novel L1 retrotransposon marker for HeLa cell line identification. Biotechniques 46: 277-284. doi:10.2144/000113089

Ramírez F, Ryan DP, Grüning B, Bhardwaj V, Kilpert F, Richter AS, Heyne S, Dündar F, Manke T. 2016. deepTools2: a next generation web server for deep-sequencing data analysis. Nucleic Acids Res 44: W160-W165. doi:10.1093/nar/gkw257

Rhee I, Jair KW, Yen RWC, Lengauer C, Herman JG, Kinzler KW, Vogelstein B, Baylin SB, Schuebel KE. 2000. CpG methylation is maintained in human cancer cells lacking DNMT1. Nature 404: 1003-1007. doi:10.1038/ 35010000

Rhee I, Bachman KE, Park BH, Jair KW, Yen RW, Schuebel KE, Cui H, Feinberg AP, Lengauer C, Kinzler KW, et al. 2002. DNMT1 and DNMT3b cooperate to silence genes in human cancer cells. Nature 416: $552-556$. doi:10.1038/416552a

Roadmap Epigenomics Consortium, Kundaje A, Meuleman W, Ernst J, Bilenky M, Yen A, Heravi-Moussavi A, Kheradpour P, Zhang Z, Wang $\mathrm{J}$, et al. 2015. Integrative analysis of 111 reference human epigenomes. Nature 518: $317-330$. doi:10.1038/nature 14248

Rodriguez JM, Maietta P, Ezkurdia I, Pietrelli A, Wesselink JJ, Lopez G, Valencia A, Tress ML. 2013. APPRIS: annotation of principal and alternative splice isoforms. Nucleic Acids Res 41: D110-D117. doi:10.1093/ nar/gks1058

Schmidl C, Rendeiro AF, Sheffield NC, Bock C. 2015. ChIPmentation: fast, robust, low-input ChIP-seq for histones and transcription factors. Nat Methods 12: 963-965. doi:10.1038/nmeth.3542

Song CX, Szulwach KE, Dai Q, Fu Y, Mao SQ, Lin L, Street C, Li Y, Poidevin $\mathrm{M}, \mathrm{Wu} \mathrm{H}$, et al. 2013. Genome-wide profiling of 5-formylcytosine reveals its roles in epigenetic priming. Cell 153: 678-691. doi:10.1016/ .cell.2013.04.001

Statham AL, Robinson MD, Song JZ, Coolen MW, Stirzaker C, Clark SJ. 2012. Bisulfite sequencing of chromatin immunoprecipitated DNA (BisChIPseq) directly informs methylation status of histone-modified DNA. Genome Res 22: 1120-1127. doi:10.1101/gr.132076.111

\section{Genome Research}

www.genome.org 
mATAC-seq: DNA methylation at accessible chromatin

Suzuki M, Liao W, Wos F, Johnston AD, DeGrazia J, Ishii J, Bloom T, Zody MC, Germer S, Greally JM. 2018. Whole-genome bisulfite sequencing with improved accuracy and cost. Genome Res 28: 1364-1371. doi:10 $.1101 /$ gr.232587.117

Thurman RE, Rynes E, Humbert R, Vierstra J, Maurano MT, Haugen E, Sheffield NC, Stergachis AB, Wang H, Vernot B, et al. 2012. The accessible chromatin landscape of the human genome. Nature 489: 75-82. doi:10.1038/nature 11232

Walter M, Teissandier A, Pérez-Palacios R, Bourc'his D. 2016. An epigenetic switch ensures transposon repression upon dynamic loss of DNA methylation in embryonic stem cells. eLife 5: e11418. doi:10.7554/eLife.11418

$\mathrm{Wu} \mathrm{H}, \mathrm{Wu}$ X, Zhang Y. 2016. Base-resolution profiling of active DNA demethylation using MAB-seq and caMAB-seq. Nat Protoc 11: 10811100. doi:10.1038/nprot.2016.069

Yin Y, Morgunova E, Jolma A, Kaasinen E, Sahu B, Khund-Sayeed S, Das PK, Kivioja T, Dave K, Zhong F, et al. 2017. Impact of cytosine methylation on DNA binding specificities of human transcription factors. Science 356: eaaj2239. doi:10.1126/science.aaj2239

Young L, Sung J, Stacey G, Masters JR. 2010. Detection of Mycoplasma in cell cultures. Nat Protoc 5: 929-934. doi:10.1038/nprot.2010.43

Yu M, Hon GC, Szulwach KE, Song CX, Zhang L, Kim A, Li X, Dai Q, Shen Y, Park B, et al. 2012. Base-resolution analysis of 5-hydroxymethylcytosine in the mammalian genome. Cell 149: 1368-1380. doi:10.1016/j.cell .2012.04.027

Yu G, Wang LG, He QY. 2015. ChIPseeker: an R/Bioconductor package for ChIP peak annotation, comparison and visualization. Bioinformatics 31: 2382-2383. doi:10.1093/bioinformatics/btv145

Zaret KS, Carroll JS. 2011. Pioneer transcription factors: establishing competence for gene expression. Genes Dev 25: 2227-2241. doi:10.1101/gad .176826 .111

Zaret KS, Mango SE. 2016. Pioneer transcription factors, chromatin dynamics, and cell fate control. Curr Opin Genet Dev 37: 76-81. doi:10.1016/j .gde.2015.12.003

Zhu LJ, Gazin C, Lawson ND, Pagès H, Lin SM, Lapointe DS, Green MR. 2010. ChIPpeakAnno: a Bioconductor package to annotate ChIP-seq and ChIP-chip data. BMC Bioinformatics 11: 237. doi:10.1186/14712105-11-237

Zilberman D, Coleman-Derr D, Ballinger T, Henikoff S. 2008. Histone H2A.Z and DNA methylation are mutually antagonistic chromatin marks. Nature 456: 125-129. doi:10.1038/nature07324

Received October 22, 2018; accepted in revised form May 1, 2019. 


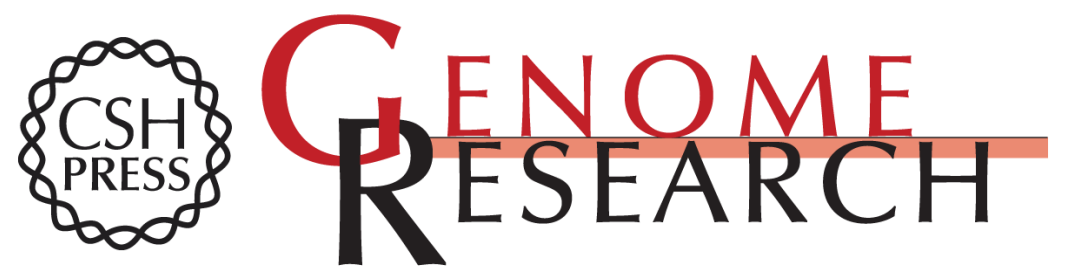

\section{methyl-ATAC-seq measures DNA methylation at accessible chromatin}

Roman Spektor, Nathaniel D. Tippens, Claudia A. Mimoso, et al.

Genome Res. 2019 29: 969-977 originally published online June 3, 2019

Access the most recent version at doi:10.1101/gr.245399.118

\section{Supplemental} Material

References

Creative

Commons

License

Email Alerting Service
http://genome.cshlp.org/content/suppl/2019/06/03/gr.245399.118.DC1

This article cites 58 articles, 12 of which can be accessed free at: http://genome.cshlp.org/content/29/6/969.full.html\#ref-list-1

This article is distributed exclusively by Cold Spring Harbor Laboratory Press for the first six months after the full-issue publication date (see

$\mathrm{http}: / / g$ enome.cshlp.org/site/misc/terms.xhtml). After six months, it is available under a Creative Commons License (Attribution-NonCommercial 4.0 International), as described at http://creativecommons.org/licenses/by-nc/4.0/.

Receive free email alerts when new articles cite this article - sign up in the box at the top right corner of the article or click here.

\section{Affordable, Accurate Sequencing.}

To subscribe to Genome Research go to:

https://genome.cshlp.org/subscriptions 